\title{
Effect of some plant oils on efficiency of Lufenuron in controlling Trogoderma granarium
}

\author{
Batool Abdullah Karso, \\ Lecturer, Plant Protection Department, Faculty of Agriculture and Forestry, $\quad$ University of Duhok, Duhok, \\ Iraq
}

\begin{abstract}
The current study aimed to test the effect of five vegetable oils, sesame, pistachio field, soybean, sunflower and almond with inhibitors' insecticide (Lufenuron), using four different mixing ratios (pesticide:vegetable oil) 1: 0.5, 1:1, 1:2, and 1: 3 and four different concentrations 50, 100, 150, and $200 \mathrm{ppm}$ for each mixing ratio, and mixture ratio in the proportion of activation, synergy and potentiation on the \% mortality of grain beetle larvae Trogoderma granarium. The study results showed that the effect of mixture Sunflower oil by mixing 2 oil : 1 pesticide highest toxicity to the revitalization of the pesticide in the grain beetle larvae of the third instar of poetry at a rate of 2.33, which was the rate of activation synergistically and potentially 1, 1.33 respectively, The sunflower oil, sesame oil and peanut oil gave a mixture with the pesticide Lufenuron by mixing oil 1: 1 ratio less toxicity pesticide antagonism reached 0.48, 0.56, 0.63 respectively.
\end{abstract}

KEYWORDS: Mixing ratio, Vegetable oil, Activation, Synergy, Potentiation, Grain beetle, Lufenuron, inhibitors' insecticide.

\section{INTRODUCTION}

The extensive and uncontrolled pesticides application has led to appearance of numerous species of resistance insect to pesticides, this reach to 460 species in 2003 ( Al Mallah and Al jubury, 2012).The side effects of adding pesticides led many environmentalists to call to stop use of pesticides preventing its production, but unfortunately, this call that unrealistic evidence that there is an increase in the production and use of pesticides in the world, which of course indication that pesticides are still the means adopted by man to control the pests. (Meister, 2010). The most realistic alternative to reduce the use of pesticides is to rationalize the use and follow the correct methods in order to reduce collateral damage, in addition to reduce the damage of side effect. The addition of doping substances of pesticides to increase their effectiveness at low concentrations and use other methods and strategies that helps in this direction. Therefore, the present study aimed to protect the environment from the negative effects of pesticides through the study of the dynamic influence of some vegetable oils In response grain beetles larvae for some modern pesticides through the study of the influence of type of oil and the mixing ratio of oil with inhibitors' insecticide as a Lufenuron pesticide in rate of activation, synergy, and potentiation of the pesticide. Determine the mechanism of activation in the Lufenuron pesticide, whether synergy or potentiation.

\section{MATERIALS ANS METHODS}

For the implementation of this study, we made three different mixing ratios (pesticide: vegetable oil) 1: 0.5, 1:1, 1: 2 , and 1: 3 respectively, five vegetable oils were used for each ratios sunflower oil, sesame oil, pistachio field oil, soybean oil, and almond oil, with inhibitors' insecticide (Lufenuron), and all mixtures have been mitigated with Acetone for different concentrations 50,100,150, and 200 ppm for each mixing ratio.

Three replication and ten grain beetle poetry larvae third instars old were treated by dowsing in each mixing ratio, and control treated with Acetone only.

All larvae placed inside Petri dishes $9 \mathrm{Cm}$ Diameter in incubator with Temperature of $30 \mathrm{C}$ and Humidity 65 $70 \%$

The results were collected after 24 hours, \% mortality calculated using equation Abbott (1925), extract values of 50 LC manner using Finney (1971), activation ratio using equation Metcalf (1972), and the interactive between the activation, synergy, and potentiation for each mixing ratio.

Synergy ratio, we excluded Relay ratio, which represents the \% mortality caused by stimulant through the following steps:

- Find a mortality rate of the corrected concentrations used for each of the oils and pesticides used in the study.

- Find a mortality rate corrected for concentrations of the mixture used (oil + pesticide). 
- Correction mortality rate of the mixture using the modified equation Abbott mentioned in the navigator Al mallah and Al-Juboury (2011), and to get rid of the deadly effect of the stimulant substance, which represents the proportion of Relay and humble is to keep the synergistic impact only, as in the equation:

$\%$ mortality corrected (pesticide $)=(\%$ mortality of a mixture $-\%$ mortality of anabolic substance)/(100- \% mortality of anabolic substance)* 100 .

- Draw lines of toxicity for the pesticide and its mixture separately using the corrected \% killing which calculated for each of the LC 50 for the pesticide and its mixture.

- Calculate the proportion of synergistic effect of using the equation Metcalf (1972), which requires modified to make it clear to Article servo toxic effect.

- Ratio of synergistic effect $=50 \mathrm{LC}$ value of the pesticide $\backslash 50 \mathrm{LC}$ value of the pesticide apron (mixture corrected).

- The total expense ratio of activation $=50 \mathrm{LC}$ value of the pesticide $\backslash 50 \mathrm{LC}$ value of the mixture.

- Calculate the proportion of oil in the Relay activated after the activation ratio was calculated and the percentage of total synergies it can be calculated using the ratio of Relay the following equation:

Relay ratio $=$ ratio of activation - the proportion of synergy.

\section{RESULTS AND DISCSSION}

\section{1- The effect of mixing ratio of Lufenuron( inhibitors' insecticide) with some oils and \% mortality in the} Trogoderma granarium larvae larvae.

The results showed that the average of \% mortality in Trogoderma granarium larvae treated with Lufenuron pesticide mixed with sunflower oil and has varied depending on the mixing ratio and the concentration used in the mixture, and mixing ratio 3:1 (oil: pesticide) gave the highest average mortality rate reaching $85 \%$ at $200 \mathrm{ppm}$, followed by the mixing ratio of $1: 0.5$ where is the $\%$ mortality $76 \%$ at the same concentration and the lowest number at a concentration of $50 \mathrm{ppm}$ by mixing 1:1 reached $14.28 \%$. And showed that the best mixing ratio is $2: 1$ which gave lower LC 50 value was 30.39 , which indicates the toxicity of the oil mixture as in Table (1).The results showed that the average \% mortality in the larval treated with a mixture sesame oil and pesticide has varied depending on the mixing ratio and the concentration used in the mixture, and the highest average $\%$ mortality was $90 \%$ when using sesame oil mixed with the pesticide by 2:1 and 3:1 (oil: pesticide), and at $200 \mathrm{ppm}$ while the lowest $\%$ mortality was $22.22 \%$ at the mixing ratio $1: 1$ at $50 \mathrm{ppm}$. Also shown that the best mixing ratio is $2: 1$, where given less value LC50 reached 58.86, which confirms the toxicity of the oil mixture as shown in Table (2). While Mohammed (2009) mentioned that the sesame oil was best oils activation with pesticide Phenam activated by $1.9 \%$ and the relative effectiveness of 47.61 and the evidence did not show the toxicity of 100 clove oil activation against $C$. maculates $\mathrm{F}$. with mixing 1:1 (pesticide: oil).

The results also show that the average of $\%$ mortality in the larval treated with a mixture peanut oil and pesticide has varied depending on the mixing ratio and the concentration of mixture, and the highest mortality rate $94 \%$ when using peanut oil mixed with the pesticides by 2:1 (oil: pesticide) at $200 \mathrm{Ppm}$, and less mortality ratio reached $28.5 \%$ at the mixing ratio $1: 1$ concentration of $50 \mathrm{ppm}$. Also shown that the best mixing ratio is $2: 1$, where given less value amounted to 44.81 L50 where the overall average of 76.75 and killed by confirming the toxicity of the oil mixture, Table (3). The results also showed that the average of $\%$ mortality in larvae with a mixture of soybean oil and pesticide has varied depending on the mixing ratio and the concentration, and mixing ratio of 3:1 (oil: pesticide) gave the highest average of mortality reached to $95 \%$ at $200 \mathrm{ppm}$ and less \% mortality at a concentration $50 \mathrm{ppm}$ by mixing $2: 1$ amounted to $42 \%$ at the concentration, and by mixing $3: 1$ as showed in Table (4). The results in the same table showed that the best Longs mixing ratio is 3:1, where given the lower value LC50 amounted to 45.36 and the \% mortality reached to $77 \%$ and this indicates that the toxicity of the oil mixture. The results also show that the average of $\%$ mortality in the larval treated with a mix Almond oil and pesticide has varied depending on the mixing ratio and the concentration, and mixing ratio of 1: 1 (oil: pesticide) gave the highest average at $90.47 \%$ at $200 \mathrm{ppm}$ the lowest rate was at a concentration of $50 \mathrm{ppm}$ by mixing $3: 1$ of $35 \%$, as showed in table (5), and the best mixing ratio is $1: 1$ which gave less value LC50 amounted to 59.27 and the average for the year amounted to $71.42 \%$ and this indicates the toxicity of the oil mixture.The results showed also that the type of oil and the mixing ratio has varying effect in the average of the larvae $\%$ mortality table (6), It was found that the highest average in the \% mortality in the larvae resulting from the interaction between the type of oil and the mixing ratio with the pesticide reached $77 \%$ when the mixing ratio of 3:1 to the mixture of soybean oil and pesticide, followed by the mixture of oil, peanut and pesticide reaching to $76.75 \%$ oil by mixing $2: 1$ pesticide, and compared to less average ratio was when treated with a mixture of sun flower oil and pesticide at mixing $1: 1$, reached to $40.47 \%$, followed by the mixture of sesame oil and pesticide by mixing $1: 1$, reaching to $47.21 \%$, this result is contrary to what the Mohammed (2009) mentioned that the sesame oil was best activating at $1.9 \%$ and 47.61 relative effectiveness and toxicity of evidence 100, while the Clove oil did not show activation, the reason for the superiority of soybean oil in 
activating the mortality rate that the density of soybean oil was high. Shahidi (2005) mentioned that the density of soybean oil ranges between 0.916 - 0.926, and viscosity range from $58.5-62.2 \mathrm{cp}$. (Shaaban and Al Mallah 1993) and (Abu Shanab, 2011) mentioned that the density of oil increased the stability on the body of the insect and thereby prevents the process of breathing insect which lied to insects dies by suffocation and thus explain the high rate of mortality to a mixture of soybean oil and pesticide. Dawood (1991) stressed also that the soybean oil and kernel dates oil, mineral oils, Thanite, and Phenobarbital with pesticide Deltametherin in studying the effect of activation of the pesticide against adult beetle cowpea South $\mathrm{C}$. maculatus, when used mixture 5:1 pesticide: oil, while the mixture of pesticide with sesame oil gave the lower activation as shown in Table(5).

\section{2- The effect of oil type and mixing ratio in the proportion of activation and synergy and potentiation in pesticide Lufenuron.}

Synergy ratios of mixtures oils and pesticide varied in our study depending on the type of oil and the mixing ratio. The results of the statistical analysis presence significant differences in the rates of activation at the level $\% 0.05$ depending on the factors, the highest synergy 1.55 gave by the mixture pesticide with peanut at mixing ratio of 2:1, and fallowed by mixture soybean and pesticide $3: 1$ reaching to 1.5 , while the less synergy ratio 0.37 appeared when mixing ratio $1: 1$ and mixture of sunflower oil as shown in table (7). The results showed also wide variation in the potentiation rate for mixtures of oils and pesticide depending on the type of oil and the rate of mixing it was found that the higher proportion 1.33 in a mixture of pesticide and sunflower oil, at the mixing ratio 1:2, while gave a mixture of the pesticide with almond oil the lowest rate 0.03 at the ration 3:1 as shown in table (8).Finally, and as the activation result is the sum of Synergy ratio and potentiation ratio, the results showed wide variation in the rates of activation of mixtures, the higher activation found in the mixture pesticide with sunflower oil where reached to 2.33 at the mixing ratio $2: 1$, while the lowest activation 0.48 rate shown at the same oil at mixing 1:1 compared to as shown in Table (9). In general, the mixing ratio 2:1 was the best, followed by the mixing ratio of 3:1, where the overall average for the activation 1.46 and 1.23 , respectively.Finally, the results of this study showed that the vegetable oils were used in this study has synergistic effect on the pesticide, and this is consistent with several studies that show the synergistic effect of the material added to a pesticide. Shaaban and Al Mallah (1993), Sun and Johnson (1960). Reported that The synergies mainly depends on the materials that may be motivating or inhibitory the enzymes and thus on the chemical composition of pesticides. O'Brien (1967). Mentioned that the increased of toxicity pesticides by adding synergies materials depends on several factors, like increased speed of entry into force of the pesticide through the body and the speed of arrival at the target sites, Wilkinson (1979). Reported that the additives materials can inhibitory the enzyme which responsible for the removal of toxic pesticides within the body of the insect, which leads to the accumulation of the active ingredient of the pesticide and the speed of the killings. Karso (2012). Confirmed that the influence of activation shown by vegetable oils in some pesticides due to the mechanism of synergism more than what is due to the mechanism potentintion, although rates of activation and antagonism shown by the results of the study did not only rely on type of oil used in the study, but had a mix proportions important role in this area, results of the study showed that the rates of increase of oil led to the activation contrast ratio and the ratio of 1:0.5 gave the best rates of activation. Also Karso and Nazar (2013) founded that the best mixture was peanut oil by mixing 3 Oil:1 pesticide toxicity to the revitalization of the pesticide in the grain beetle larvae of the third instar of poetry at a rate of 1.62, which was the rate of activation synergistically1.57, The sesame oil gave a mixture with the insecticide Alpha-Cypermtherin by mixing oil 2: 1 ratio less insecticide antagonism reached 0.15 .

Table (1):The effect of Sunflower oil and Match insecticides mixture concentrations of mixture ratio on the Mortality percentage of grain beetle larvae.

\begin{tabular}{|c|c|c|c|c|}
\hline Mixture ratio & Concentrate/ppm & $\%$ mortality & LC50 & Mean \\
\hline \multirow{4}{*}{$1: 0.5$} & 50 & 42 & \multirow{4}{*}{71.43} & \multirow{4}{*}{60.5} \\
\hline & 100 & 57 & & \\
\hline & 150 & 67 & & \\
\hline & 200 & 76 & & \\
\hline \multirow{4}{*}{$1: 1$} & 50 & 14.28 & \multirow{4}{*}{146} & \multirow{4}{*}{40.47} \\
\hline & 100 & 28.85 & & \\
\hline & 150 & 57.41 & & \\
\hline & 200 & 61.9 & & \\
\hline \multirow{4}{*}{$1: 2$} & 50 & 57 & \multirow{4}{*}{30.39} & \multirow{4}{*}{66.25} \\
\hline & 100 & 70 & & \\
\hline & 150 & 67 & & \\
\hline & 200 & 71 & & \\
\hline \multirow{4}{*}{$1: 3$} & 50 & 42 & \multirow{4}{*}{69.86} & \multirow{4}{*}{63.75} \\
\hline & 100 & 57 & & \\
\hline & 150 & 71 & & \\
\hline & 200 & 85 & & \\
\hline
\end{tabular}


Table (2): Illustrated the effect of Sesame oil and Lufenuron insecticides mixture concentrations of mixture ratio on the Mortality percentage of grain beetle larvae.

\begin{tabular}{|c|c|c|c|c|}
\hline Mixture ratio & Concentrate/ppm & $\%$ mortality & LC50 & Mean \\
\hline \multirow{4}{*}{$1: 0.5$} & 50 & 28 & \multirow{4}{*}{86.97} & \multirow{4}{*}{59.5} \\
\hline & 100 & 55 & & \\
\hline & 150 & 70 & & \\
\hline & 200 & 85 & & \\
\hline \multirow{4}{*}{$1: 1$} & 50 & 22.22 & \multirow{4}{*}{122.38} & \multirow{4}{*}{47.21} \\
\hline & 100 & 44.44 & & \\
\hline & 150 & 55.5 & & \\
\hline & 200 & 66.7 & & \\
\hline \multirow{4}{*}{$1: 2$} & 50 & 43 & \multirow{4}{*}{58.86} & \multirow{4}{*}{72.5} \\
\hline & 100 & 71 & & \\
\hline & 150 & 86 & & \\
\hline & 200 & 90 & & \\
\hline \multirow{4}{*}{$1: 3$} & 50 & 43 & \multirow{4}{*}{61.77} & \multirow{4}{*}{69.5} \\
\hline & 100 & 67 & & \\
\hline & 150 & 78 & & \\
\hline & 200 & 90 & & \\
\hline
\end{tabular}

Table (3): T the effect of Peanut oil and Lufenuron insecticides mixture concentrations of mixture ratio on the Mortality percentage of grain beetle larvae.

\begin{tabular}{|c|c|c|c|c|}
\hline Mixture ratio & Concentrate/ppm & $\%$ mortality & LC50 & Mean \\
\hline \multirow{4}{*}{$1: 0.5$} & 50 & 42 & \multirow{4}{*}{49.8} & \multirow{4}{*}{65} \\
\hline & 100 & 67 & & \\
\hline & 150 & 71 & & \\
\hline & 200 & 90 & & \\
\hline \multirow{4}{*}{$1: 1$} & 50 & 28.5 & \multirow{4}{*}{112.69} & \multirow{4}{*}{49.96} \\
\hline & 100 & 42.85 & & \\
\hline & 150 & 57.12 & & \\
\hline & 200 & 71.4 & & \\
\hline \multirow{4}{*}{$1: 2$} & 50 & 57 & \multirow{4}{*}{44.81} & \multirow{4}{*}{76.75} \\
\hline & 100 & 71 & & \\
\hline & 150 & 85 & & \\
\hline & 200 & 94 & & \\
\hline \multirow{4}{*}{$1: 3$} & 50 & 40 & \multirow{4}{*}{66.83} & \multirow{4}{*}{64.75} \\
\hline & 100 & 59 & & \\
\hline & 150 & 70 & & \\
\hline & 200 & 90 & & \\
\hline
\end{tabular}

Table (4): The effect of Soybean oil and Lufenuron insecticides mixture concentrations of mixture ratio on the Mortality percentage of grain beetle larvae.

\begin{tabular}{|c|c|c|c|c|}
\hline Mixture ratio & Concentrate/nnm & of mortality & I C50 & Mean \\
\hline \multirow{4}{*}{$1: 0.5$} & 50 & 52 & \multirow{4}{*}{51.75} & \multirow{4}{*}{65.5} \\
\hline & 100 & 59 & & \\
\hline & 150 & 71 & & \\
\hline & 200 & 80 & & \\
\hline \multirow{4}{*}{$1: 1$} & 50 & 42.8 & \multirow{4}{*}{70} & \multirow{4}{*}{64.25} \\
\hline & 100 & 57.1 & & \\
\hline & 150 & 71.4 & & \\
\hline & 200 & 85.7 & & \\
\hline \multirow{4}{*}{$1: 2$} & 50 & 42 & \multirow{4}{*}{59.8} & \multirow{4}{*}{70} \\
\hline & 100 & 71 & & \\
\hline & 150 & 81 & & \\
\hline & 200 & 86 & & \\
\hline \multirow{4}{*}{$1: 3$} & 50 & 57 & \multirow{4}{*}{45.36} & \multirow{4}{*}{77} \\
\hline & 100 & 71 & & \\
\hline & 150 & 85 & & \\
\hline & 200 & 95 & & \\
\hline
\end{tabular}


Table (5): The effect of Almond oil and Lufenuron insecticides mixture concentrations of mixture ratio

\begin{tabular}{|c|c|c|c|c|}
\hline Mixture ratio & Concentrate/ppm & $\%$ mortality & LC50 & Mean \\
\hline \multirow{4}{*}{$1: 0.5$} & 50 & 42 & \multirow{4}{*}{69.93} & \multirow{4}{*}{62.5} \\
\hline & 100 & 57 & & \\
\hline & 150 & 71 & & \\
\hline & 200 & 80 & & \\
\hline \multirow{4}{*}{$1: 1$} & 50 & 42.85 & \multirow{4}{*}{59.27} & \multirow{4}{*}{71.42} \\
\hline & 100 & 71.42 & & \\
\hline & 150 & 80.95 & & \\
\hline & 200 & 90.47 & & \\
\hline \multirow{4}{*}{$1: 2$} & 50 & 43 & \multirow{4}{*}{81.9} & \multirow{4}{*}{54.25} \\
\hline & 100 & 53 & & \\
\hline & 150 & 58 & & \\
\hline & 200 & 63 & & \\
\hline \multirow{4}{*}{$1: 3$} & 50 & 35 & \multirow{4}{*}{79.66} & \multirow{4}{*}{56.25} \\
\hline & 100 & 50 & & \\
\hline & 150 & 60 & & \\
\hline & 200 & 60 & & \\
\hline
\end{tabular}

Table (6): The effect of oil type mixed with Inhibitor insecticide(Lufenuron) pesticide on the mortality of grain beetle larvae.

\begin{tabular}{|c|c|c|c|c|}
\hline \multirow[t]{2}{*}{ Oil Type } & \multicolumn{4}{|c|}{ Mixture ratio ( pesticide : oil ) } \\
\hline & $1: 0.5$ & $1: 1$ & $1: 2$ & $1: 3$ \\
\hline Sunflower & 60.50 & 40.47 & 66.25 & 63.75 \\
\hline Sesame & 59.50 & 47.21 & 72.50 & 69.50 \\
\hline Peanut & 65.00 & 49.96 & 76.70 & 64.75 \\
\hline Soybean & 65.50 & 64.25 & 70.00 & 77.00 \\
\hline Almond & 62.50 & 71.42 & 54.25 & 56.25 \\
\hline
\end{tabular}

Table (7): Effect of vegetables oils and pesticide mixture ratio on the Synergism on Grain beetle larvae.

\begin{tabular}{|c|c|c|c|c|}
\hline \multirow{2}{*}{ Type of oil } & \multicolumn{4}{|c|}{ Mixing ratio } \\
\cline { 2 - 5 } & $1: 0.5$ & $1: 1$ & $1: 2$ & $1: 3$ \\
\hline Sunflower & 0.69 & 0.37 & 1.00 & 0.81 \\
\hline Sesame & 0.45 & 0.45 & 1.07 & 0.80 \\
\hline Peanut & 1.27 & 0.51 & 1.55 & 0.99 \\
\hline Soybean & 1.20 & 1.09 & 1.14 & 1.50 \\
\hline Almond & 0.90 & 0.95 & 0.54 & 0.86 \\
\hline Mean & 1.38 & 0.96 & 1.02 & 1.24 \\
\hline
\end{tabular}

Table (8): Effect of vegetables oils and pesticide mixture ratio on the Potentation on Grain beetle larvae.

\begin{tabular}{|c|c|c|c|c|}
\hline \multirow{2}{*}{ Type of oil } & \multicolumn{4}{|c|}{ Mixing ratio } \\
\cline { 2 - 5 } & $1: 0.5$ & $1: 1$ & $1: 2$ & $1: 3$ \\
\hline Sunflower & 0.30 & 0.11 & 1.33 & 0.20 \\
\hline Sesame & 0.36 & 0.13 & 0.13 & 0.70 \\
\hline Peanut & 0.15 & 0.12 & 0.10 & 0.16 \\
\hline Soybean & 0.20 & 0.06 & 0.10 & 0.20 \\
\hline Almond & 0.11 & 0.11 & 0.40 & 0.03 \\
\hline Mean & 0.14 & 0.27 & 0.27 & 0.18 \\
\hline
\end{tabular}

Table (9): Effect of vegetables oils and pesticide mixture ratio on the activation on Grain beetle larvae.

\begin{tabular}{|c|c|c|c|c|}
\hline \multirow{2}{*}{ Type of oil } & \multicolumn{4}{|c|}{ Mixing ratio } \\
\cline { 2 - 5 } & $1: 0.5$ & $1: 1$ & $1: 2$ & $1: 3$ \\
\hline Sunflower & $1: 00$ & 0.48 & 2.33 & 1.01 \\
\hline Sesame & 0.81 & 0.57 & 1.20 & 1.50 \\
\hline Peanut & 1.42 & 0.63 & 1.60 & 1.06 \\
\hline Soybean & 1.40 & 1.01 & 1.20 & 1.70 \\
\hline Almond & 1.01 & 1.20 & $1: 00$ & 0.89 \\
\hline Mean & 1.51 & 1.20 & 1.26 & 1.41 \\
\hline
\end{tabular}


[1]. Abbot, W. S. I. (1925). A method for computing the effectiveness on pesticides. Journal of Economic Entomology. 18:265 - 267.

[2]. Al Mallah N. M. and Al-jubury A.Yonis. (2011).A New Method for Calculating the Synergism and Potentiation Percentage of Pesticides Activator Compounds. Mesopotamia Journal of Agriculture. 39(4)244-249.

[3]. Al Mallah N. M. and Al-jubury A.Yonis. (2012). Chemical Pesticides, Classification, Mode of Action and Metabolism. (2014) Al-Yazori for Scientific Publishing, Amman, Jordan pp. 213-217.

[4]. Daoud, A. S. (1991). Synergistic effect of some vegetable and mineral oil on the Deltamethrin pesticide against adult Callosobruchus maculates (f.) Coleoptera, Bruchidae. Mesopotamia journal of agriculture. Vol (23) 1:245-250.

[5]. Finney, J.C. (1952). Probit Analysis Cambridge University, Press London, 256 Pp.

[6]. Karso, B. A. (2012). The effect of some vegetable oils and using methods on responding of Khapra larvae to some pesticides, $\mathrm{PhD}$ thesis, University of Mosul, Iraq. pp 131.

[7]. Karso, B. A. and N. M. Al- Mallah (2013). The effected of mixting ratios some vegetable oils in synergism and potation the AlCypermathrin insecticide in grain beetles . Mesopotamia Journal of Agriculture. 41(3)274-285.

[8]. Meister, R.T. (2010). Crop Protection of Handbook. Wilaughby, OH. USA.

[9]. Metcalf, R.L. (1972). Mode of action of pesticide synergist. Ann. Rev. Entomology. 12: $225-229$.

[10]. Mohamed, H. A. K. (2009). Effect of sesame oil, sweet almond oil, and glove oil on the efficiency of some Pesticide to cow pea weevil Callosobruchus maculates (fab,) (Coleoptera: Bruchidae). Journal of Tikrit for Agricultural Sciences Vol (9) 1: 268-278.

[11]. O’Brien, R. D. (1967). Pesticides action and metabolism. Academic press, New York, London, Pp. $164-220$

[12]. Shaban Awwad and N. M. Al Mallah (1993). Pesticides, Book,Mosul, Iraq, pp 520.

[13]. Shahidi, Fereidon (2005). Vegetable oils, Baileys Industrial oil and Fat products .Vol: (6).

[14]. Sun, Y.P. and E.R. Johnson. (1960). Synergistic and antagonistic actions pesticide - Synergist combinations and their mode of action. Journal of Agricultural Food Chemistry. 8 (4): 261 - 266

[15]. Wilkinson, C.F. (1979). The use of insect sub cellular components for studying the metabolism of xenobiotics. In (Paulson, G.D., D.S. Fear, and E.P. Marks eds ) Xenobiotic metabolism in vitro method . ACS, Washington, Pp. $249-284$. 